\title{
A numerical simulation of natural convection in an isosceles triangular enclosure heated from below
}

\author{
Chengwang Lei ${ }^{1} \quad$ John C. Patterson ${ }^{2}$
}

(Received 27 August 2006; revised 14 December 2007)

\begin{abstract}
We explore the transient natural convection in an isosceles triangular enclosure subject to cooling at the inclined surfaces and simultaneous heating at the base. The problem is numerically simulated using a commercial CFD package. The flow parameters considered here correspond to those adopted in a previous experiment. The study shows that the transient flow development in an isosceles triangular enclosure undergoes an early stage, a transitional stage and a quasi-steady stage for the present Grashof numbers. The transition of the flow from symmetric structures to asymmetric structures is observed. The good agreement of the present numerical results with previously reported experiments suggests that the present numerical model is applicable to similar problems over a broad range of parameters.
\end{abstract}

See http://anziamj.austms.org.au/ojs/index.php/ANZIAMJ/article/view/100 for this article, (c) Austral. Mathematical Soc. 2007. Published December 19, 2007. ISSN 1446-8735 


\section{Contents}

1 Introduction

C631

2 Numerical details

C633

3 Results and discussion

C635

3.1 Flow response at $\mathrm{Gr}=2.36 \times 10^{5} \ldots \ldots \ldots \ldots$ C635

3.2 Heat transfer at $\mathrm{Gr}=2.36 \times 10^{5} \ldots \ldots \ldots \ldots$ C639

3.3 Results for different Grashof numbers . . . . . . . . . . . C639

4 Conclusions

C640

References

C642

\section{Introduction}

An isosceles triangular enclosure is an appropriate model of typical attics of buildings. In real life situations, the temperature on the roof (inclined surfaces) varies with the outside conditions, whereas the temperature on the base (horizontal surface) depends on the inside conditions. In a typical diurnal cycle, the absorption of solar radiation during the daytime results in a higher temperature on the roof than that on the base, and the cooling by the atmosphere during the night-time results in a lower temperature on the roof. Under both the daytime and night-time conditions, the temperature differences on the boundaries cause a spatial variation of the air density inside the enclosure, which in turn initiates natural convection within the attic space.

Heat transfer through the attics of buildings, which is mainly governed by natural convection, contributes significantly to the heat capture or loss of buildings. This motivated a series of experimental and theoretical investi- 
gations over the last three decades [1, 2, 3, 4]. Most of the previous studies are concerned with the steady-state flow and heat transfer under constant heating or cooling conditions [1,3], which are not relevant to the unsteady nature of the thermal forcing. Only a few studies targeted the unsteady problem in the attic space $[2,5]$, and limited features of the unsteady flow have been revealed from those studies. Moreover, early numerical and experimental studies of the attic problem generally assumed symmetric flow structures about the geometric symmetry plane [6, 7]. However, the recent study of Holtzman et al. [8] conducted in a full isosceles triangular domain under night-time conditions revealed that the natural convection flow in an attic undergoes a transition from symmetric to asymmetric structures as the Grashof number increases. The claim made by Holtzman et al. [8] was mainly based on numerical simulations of the steady state flow in attics with a range of aspect ratios and Grashof numbers. Holtzman et al. [8] also conducted a simple flow visualization experiment by slowly injecting smoke into the enclosure, and the experiment qualitatively supported their numerical results.

In order to confirm the validity of the claim with regard to the symmetry of the flow in an isosceles triangular enclosure, Lei et al. [9] conducted a series of experiments employing a shadowgraph technique to visualize the transient natural convection. The experiments were carried out for a fixed aspect ratio and a range of Grashof numbers beyond the critical Grashof number for the transition from symmetric to asymmetric flow structures reported by Holtzman et al. [8]. Many interesting features of the transient flow development have been unveiled by Lei et al. [9].

The present study is the corresponding numerical simulation of the flow visualization experiment reported by Lei et al. [9]. The transient flow in an isosceles triangular enclosure subject to the night-time conditions is numerically calculated for different Grashof numbers using a commercial Computational Fluid Dynamics (CFD) package. Both qualitative and quantitative results are presented. 


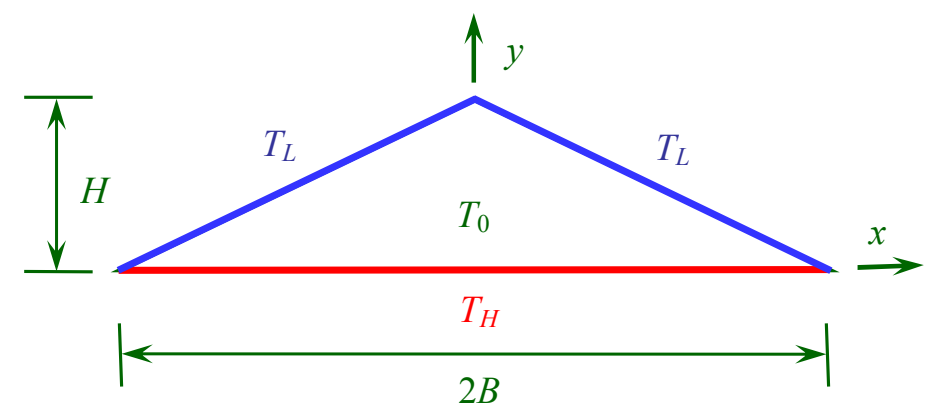

Figure 1: Schematics of the numerical model.

\section{$2 \quad$ Numerical details}

The flow domain and the coordinate system adopted in the present numerical simulation are shown in Figure 1. The dimensions of the isosceles triangle correspond to those of the experimental model adopted by Lei et al. [9], which has a height of $H=24 \mathrm{~mm}$ and a base length of $2 B=96 \mathrm{~mm}$. These dimensions were chosen so that the range of the Grashof numbers examined by Holtzman et al. [8], which covered the critical Grashof number for the transition from symmetric to asymmetric flows, is achieved with this model. Due to the difficulties in the shadowgraph flow visualization with air, the experiments reported by Lei et al. [9] were conducted with water as the medium. Accordingly, in the present numerical model, the enclosure is filled with water, which has an initial temperature of $T_{0}$. At the time $t=0$, the two inclined surfaces and the base of the enclosure are subject to instantaneous cooling and heating at constant temperatures $T_{L}\left(<T_{0}\right)$ and $T_{H}\left(>T_{0}\right)$ respectively.

Heat is then transferred from the base through the enclosure to the inclined surfaces. The heat transfer through the enclosure depends on the flow and temperature structures resulting from natural convection, which are described by the usual continuity, momentum and energy equations. Boussi- 
TABLE 1: Flow parameters for the present simulations $(A=0.5)$.

\begin{tabular}{ccccccc}
\hline No & $T_{0}$ & $T_{H}$ & $T_{L}$ & $\Delta T$ & $\operatorname{Pr}$ & $\mathrm{Gr}$ \\
\hline 1 & & $22.5^{\circ} \mathrm{C}$ & $18.5^{\circ} \mathrm{C}$ & $4^{\circ} \mathrm{C}$ & & $1.18 \times 10^{5}$ \\
2 & $20.5^{\circ} \mathrm{C}$ & $24.5^{\circ} \mathrm{C}$ & $16.5^{\circ} \mathrm{C}$ & $8^{\circ} \mathrm{C}$ & 7.06 & $2.36 \times 10^{5}$ \\
3 & & $28.5^{\circ} \mathrm{C}$ & $12.5^{\circ} \mathrm{C}$ & $16^{\circ} \mathrm{C}$ & & $4.73 \times 10^{5}$ \\
\hline
\end{tabular}

nesq assumptions are made for the water in this study, and no slip and rigid conditions are assumed for all the interior surfaces. The natural convection in the enclosure is controlled by three dimensionless parameters. These are the aspect ratio $A$, the Prandtl number Pr and the Grashof number Gr:

$$
A=\frac{H}{B} ; \quad \operatorname{Pr}=\frac{\nu}{\kappa} ; \quad \mathrm{Gr}=\frac{g \beta \Delta T H^{3}}{\nu^{2}} ;
$$

where $g$ is the acceleration due to gravity, $\beta$ is the coefficient of thermal expansion, $\nu$ is the kinematic viscosity, $\kappa$ is the thermal diffusivity, and $\Delta T$ is the temperature difference between the base and inclined surfaces, that is, $\Delta T=T_{H}-T_{L}$. All the fluid properties are evaluated at the reference temperature $T_{0}$.

In this study, the aspect ratio of the enclosure is fixed at $A=0.5$. We consider three cases corresponding to three out of the five experiments reported by Lei et al. [9]. The flow parameters are listed in Table 1.

The complete set of governing equations along with the velocity and thermal boundary conditions are solved using the commercial CFD package Fluent 6.2. A segregated solver is adopted with a second order implicit scheme. The SIMPLE method is selected for coupling pressure and velocity, and the spatial discretization is done by a second order upwind scheme. In order to avoid the singularity at the intersection points between the roofs and the base, the tips there are cut by approximately $4 \%$, and adiabatic and no-slip rigid walls are added at the cutting points. The remaining physical domain is then discretized using a structured mesh. Mesh and time step dependence tests have been conducted based on three meshes with three corresponding 
time steps. Based on these tests, a mesh with 16,000 elements and a time step of $0.05 \mathrm{~s}$ are adopted for the present simulations.

\section{Results and discussion}

We consider the transient flow response in the isosceles triangular enclosure to the abrupt heating and cooling through the boundaries. With the three sets of simulations conducted in this study, very similar flow features are observed. In this section, the overall features of the flow development in the isosceles triangular enclosure are discussed based on the results obtained with a medium temperature difference of $8^{\circ} \mathrm{C}$, corresponding to a Grashof number of $\mathrm{Gr}=2.36 \times 10^{5}$. The comparisons of the results for different Grashof numbers are also discussed.

\subsection{Flow response at $\mathrm{Gr}=2.36 \times 10^{5}$}

The transient development of the flow in the enclosure is roughly classified into three stages: an early stage, a transitional stage and a quasi-steady stage. Each of the three stages of the flow development is described below.

Early stage flow As soon as the heating and cooling are imposed on the boundaries, heat is conducted into the interior fluid from the base and out of the interior fluid through the inclined surfaces. As a consequence, thermal boundary layers develop adjacent to the interior surfaces. For the night-time cooling case considered in this study, there are two cooling thermal boundary layers, one along each inclined surface, and one heating thermal boundary layer above the base.

The development of the thermal boundary layers is observed in Figure 2, 

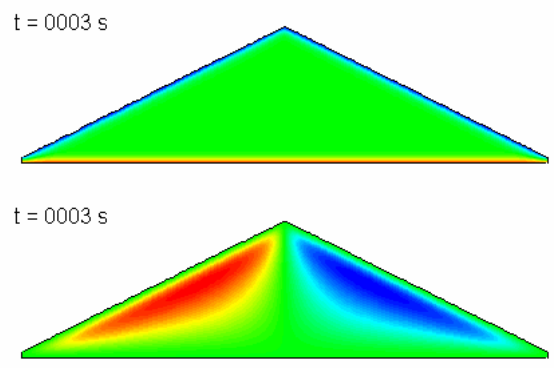

(a)
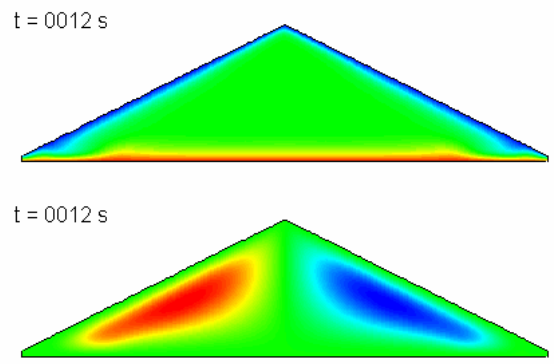

(c)
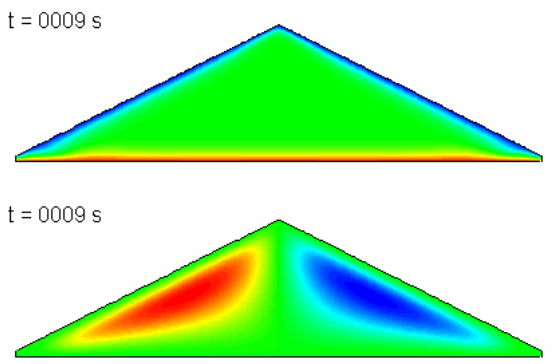

(b)
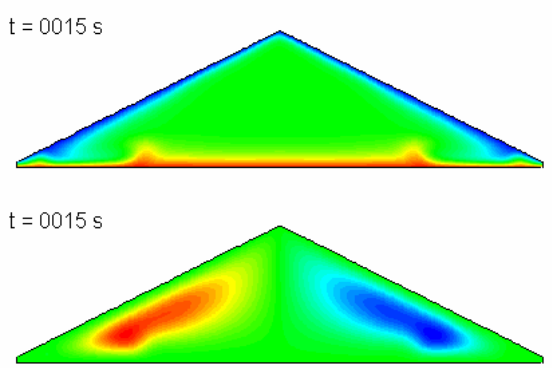

(d)

FiguRE 2: Early-stage flow $\left(\mathrm{Gr}=2.36 \times 10^{5}\right)$. Upper: isotherms; Lower: stream functions.

which shows the contours of the isotherms and stream functions at several time instances during the early stage of the flow development. In the isotherm plots, the green background represents the interior fluid; the light and dark blue bands adjacent to the inclined surfaces represent the cooling thermal boundary layers; and the red and yellow band above the base represents the heating thermal boundary layer. The growth of all the three thermal boundary layers with time is clearly seen from the isotherms in Figure 2.

While the cooling thermal boundary layers adjacent to the inclined surfaces are growing, the buoyancy effect results in two downward gravity currents, one along each inclined surface. The downward gravity currents change 
their directions after they reach the bottom tips of the enclosure, and subsequently form two opposing horizontal streams along the base and toward the center (indicated by the horizontal shift of the two bump-like structures in the heating thermal boundary layer in Figures 2(c) and 2(d). The two horizontal streams then meet and rise up at the center of the enclosure. As a consequence, two primary circulation cells are formed, one on each side of the geometric symmetry plane (refer to the contours of stream functions in Figure 2). The flow is symmetric at this stage.

Transitional stage flow The transitional stage flow is characterized by the appearance of convective instabilities. On one hand, the heating through the base of the enclosure results in a temperature structure in the horizontal boundary layer which is potentially unstable to Rayleigh-Benard instabilities. When a critical condition is met, the instability manifests in the form of rising hot water plumes, as seen in Figure 3(a). Due to the existence of a horizontal flow along the base, the bump-like structure associated with the rising hot water plume travels toward the center of the enclosure, giving a traveling wave-like appearance. On the other hand, the cooling through the inclined surfaces also results in potentially unstable thermal boundary layers along the surfaces. The instability appears in the form of sinking cold water plumes seen in Figure 3(b).

The subsequent development of the flow in the enclosure is dominated by the occurrence of convective instabilities. Two major changes to the instability features are noticeable in Figure 3. First, the wave number of the thermal instability at $t=50 \mathrm{~s}$ is larger than that at $t=20 \mathrm{~s}$, and it remains approximately of the same order for the rest of the flow development. Second, the thermal instability becomes weaker at $t=50 \mathrm{~s}$ compared with that at $t=20 \mathrm{~s}$. Due to the occurrence of the thermal instabilities in the forms of rising and sinking plumes, cellular structures are formed on both sides of the symmetry plane. However, Figure 3 shows that the overall flow structure remains symmetric at this stage. 


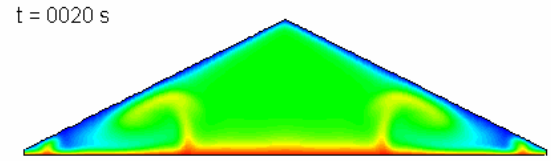

$t=0020 s$

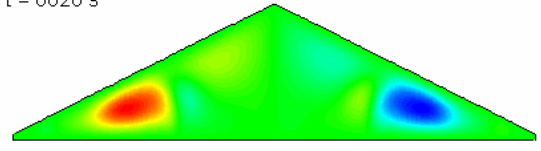

(a)
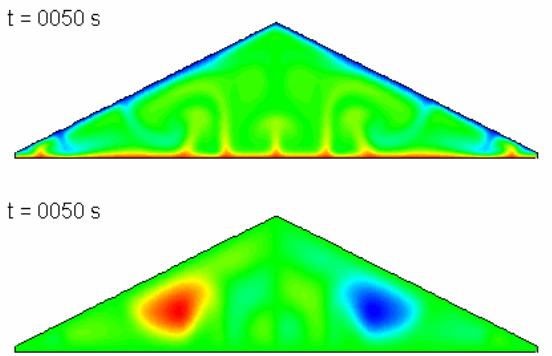

(b)

Figure 3: Transitional flow $\left(\mathrm{Gr}=2.36 \times 10^{5}\right)$ : Upper, isotherms; Lower, stream functions.

Quasi-steady stage flow Toward the end of the transitional stage, a pitchfork bifurcation starts to manifest which may due to the growth of the local Grashof number in the thermal boundary layers. As a consequence, an asymmetric flow structure is formed. An interesting phenomenon observed from the present simulation is that the convective instabilities occur alternatively on the left and right sides of the enclosure, and the horizontally moving hot water plumes from the two sides of the enclosure arrive at the center at alternating times. As a consequence, the upwelling flow near the center of the enclosure oscillates about the geometric symmetry plane. However, the time averaged flow remains symmetric about the geometric symmetry plane.

The quasi-steady state flow is characterized by the flow structure formed at the end of the transitional stage. Apart from the alternating feature of the flow described above, all other features of the flow and instabilities remain the same as those at the transitional stage. Figure 4 shows the typical temperature and flow structures at the quasi-steady state. These structures persist as long as the present simulation lasts (up to 2000 seconds). 

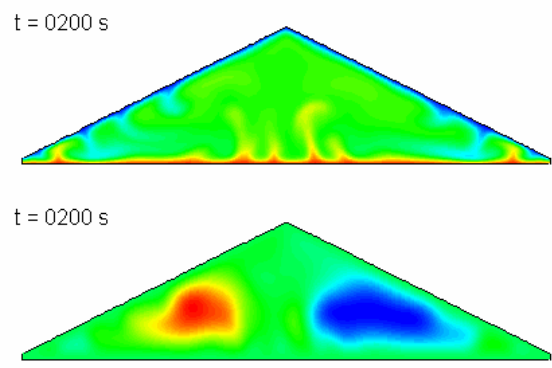

(a)
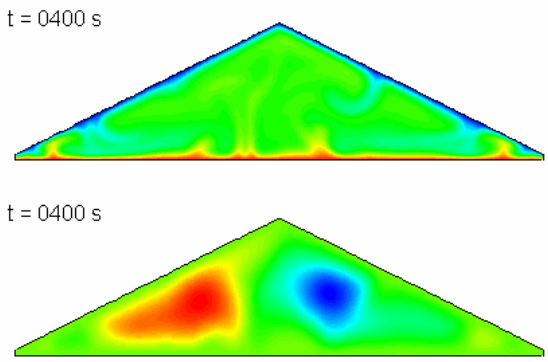

(b)

Figure 4: Quasi-steady flow $\left(\mathrm{Gr}=2.36 \times 10^{5}\right)$ : Upper, isotherms; Lower, stream functions.

\subsection{Heat transfer at $\mathbf{G r}=2.36 \times 10^{5}$}

The time histories of the calculated mean Nusselt numbers across the two inclined surfaces are plotted in Figure 5. The time histories show approximately three stages of the flow development (as roughly indicated in the figure), which are consistent with the above classification of the transient flow development. This figure also shows that the calculated mean Nusselt numbers for the two inclined surfaces are the same in the early stage and most of the transitional stage, but start to diverge toward the end of the transitional stage, confirming the previously described transition from symmetric to asymmetric flow in the enclosure.

\subsection{Results for different Grashof numbers}

The present simulation reveals similar flow features for all the three Grashof numbers considered in this study. For all the three cases, the flow development is approximately classified into an early stage, a transitional stage and a quasi-steady stage, and the characteristics of the flow at each stage are 


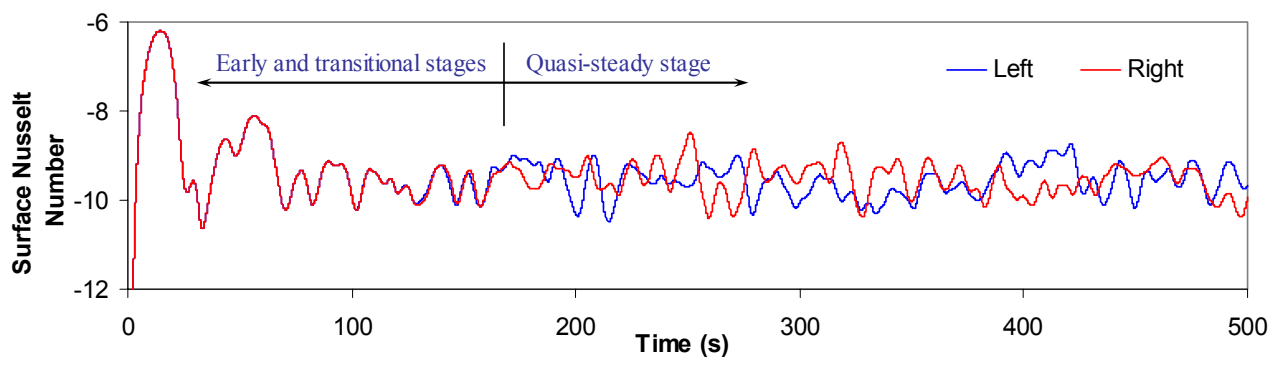

FiguRE 5: Time history of the mean Nusselt numbers of the inclined surfaces $\left(\mathrm{Gr}=2.36 \times 10^{5}\right)$.

similar for all Grashof numbers.

Figure 6 shows the typical quasi-steady state temperature and flow structures obtained for the other two cases. Comparisons of the numerical results for different Grashof numbers reveal certain variations of the flow features with the Grashof number. On one hand, the primary circulations become stronger as the Grashof number increases. On the other hand, Figure 6 shows that the convective instabilities intensify and the wave number increases with the Grashof number. This figure also shows that, at the quasi-steady state, the unstable region of the cooling thermal boundary layer expands from the lower tip regions toward the apex of the triangular domain as the Grashof number increases.

\section{Conclusions}

The transient natural convection flow in an isosceles triangular enclosure subject to abrupt heating from the base and simultaneous cooling from the inclined surfaces is investigated numerically. The simulations are conducted for a fixed aspect ratio and three different Grashof numbers ranging from 


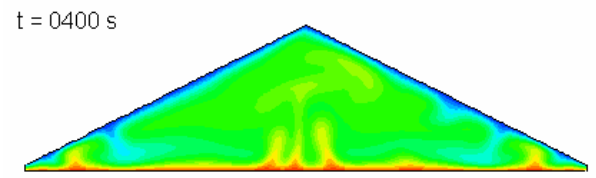

$t=0400 s$

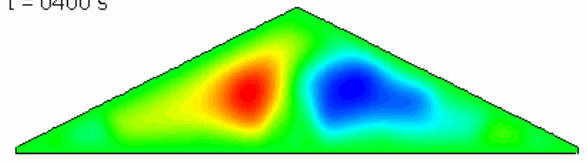

(a) $\mathrm{Gr}=1.18 \times 10^{5}$

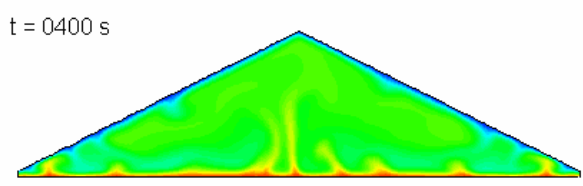

$\mathrm{t}=0400 \mathrm{~s}$

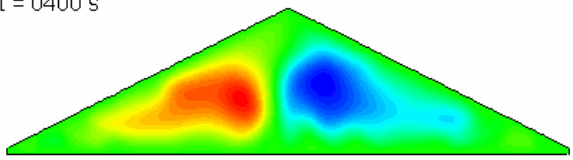

(b) $\mathrm{Gr}=4.73 \times 10^{5}$

Figure 6: Quasi-steady flow at different Grashof numbers: Upper, isotherms; Lower, stream functions.

$1.18 \times 10^{5}$ to $4.73 \times 10^{5}$. The numerical results have shown consistent flow features with the shadowgraph flow visualization reported by Lei et al. [9].

In brief, the transient flow development in the isosceles triangular enclosure due to abrupt heating and cooling through the boundaries is classified into three distinct stages, an early stage, a transitional stage, and a quasisteady stage. The early stage flow is characterized by the growth of thermal boundary layers and the initiation of primary circulations. The transitional stage flow is characterized by the appearance of convective instabilities in the form of rising and sinking thermals and the formation of cellular flow structures. The quasi-steady flow is characterized by the alternating occurrence of convective instabilities from the two sides of the enclosure and the oscillation of the upwelling flow near the center.

While the present numerical simulations and the previous experiments [9] have both confirmed the occurrence of asymmetric flow structures at the quasi-steady stage of the flow development, they have showed different features from those reported by Holtzman et al. [8]. The variation may be attributed to the different fluid media adopted in these investigations (air is adopted by Holtzman et al. [8], whereas water is adopted by Lei et al. [9] and 
in the present study). In the case with air as the medium, the mean flow at the steady state consists of a dominant circulating cell located at the center of the isosceles triangular enclosure, with additional smaller cells located on both sides of the dominant cell [8]. However, in the case with water as the medium, the time averaged flow remains approximately symmetric about the geometric symmetry plane despite the asymmetry of the instantaneous flows.

Acknowledgments This research was supported by James Cook University and the Australian Research Council.

\section{References}

[1] Flack, R. D., The experimental measurement of natural convection heat transfer in triangular enclosures heated or cooled from below, Journal of Heat Transfer, Transactions of the ASME, 102, 1980, 770-772. C632

[2] Karyakin, Y. E., Sokovishin, Y. A., Martynenko, O. G., Transient natural convection in triangular enclosures. International Journal of Heat and Mass Transfer, 31, 1988, 1759-1766.

doi:10.1016/0017-9310(88)90190-1 C632

[3] Asan, H., Namli, L., Numerical simulation of buoyant flow in a roof of triangular cross-section under winter day boundary conditions, Energy E Buildings, 33, 2001, 753-757. doi:10.1016/S0378-7788(01)00063-9 C632

[4] Haese, P. M., Teubner, M. D., Heat exchange in an attic space, International Journal of Heat and Mass Transfer, 45, 2002, 4925-4936. doi:10.1016/S0017-9310(02)00208-9 C632 
[5] Poulikakos, D., Bejan, A., The fluid dynamics of an attic space, Journal of Fluid Mechanics, 131, 1983, 251-269. doi:10.1017/S0022112083001317 C632

[6] Akinsete, V. A., Coleman, T. A., Heat transfer by steady laminar free convection in triangular enclosures, International Journal of Heat and Mass Transfer, 25, 1982, 991-998. doi:10.1016/0017-9310(82)90074-6 C632

[7] Poulikakos, D., Bejan, A., Natural convection experiments in a triangular enclosure, Journal of Heat Transfer, Transactions of the ASME, 105, 1983, 652-655. C632

[8] Holtzman, G. A., Hill, R. W., Ball, K. S., Laminar natural convection in isosceles triangular enclosures heated from below and symmetrically cooled from above, Journal of Heat Transfer, Transactions of the ASME, 122, 2000, 485-491. doi:10.1115/1.128870 C632, C633, C641, C642

[9] Lei, C., Xu, F., Patterson, J. C., Visualisation of natural convection in an isosceles triangular enclosure heated from below, Proceedings of the 5th Pacific Symposium on Flow Visualisation and Image Processing (CD-ROM), edited by B. E. Milton, Paper No. PSFVIP-5-229, 2005. C632, C633, C634, C641 


\section{Author addresses}

1. Chengwang Lei, James Cook University, School of Engineering, Townsville, Queensland 4811, Australia. mailto: Chengwang. Lei@jcu.edu.au

2. John C. Patterson, James Cook University, School of Engineering, Townsville, Queensland 4811, Australia. 